This is the peer reviewed version of the following article:

J. Simmchen, A. Baeza, A. Miguel-Lopez, M. M. Stanton, M. Vallet-Regi, D. Ruiz-Molina, S. Sánchez. Dynamics of Novel Photoactive $\mathrm{AgCl}$ Microstars and Their Environmental Applications. ChemNanoMat, (2017). 3. : 65 - . 10.1002/cnma.201600300,

which has been published in final form at https: //dx.doi.org/10.1002/cnma.201600300. This article may be used for non-commercial purposes in accordance with Wiley Terms and Conditions for Use of Self-Archived Versions. 


\title{
Dynamics of Novel Photoactive AgCl Microstars and Their Environmental Applications
}

\author{
Juliane Simmchen, ${ }^{*[a, b]}$ Alejandro Baeza, ${ }^{[c, d]}$ Albert Miguel-Lopez, ${ }^{[a,}{ }^{f]}$ Morgan M. Stanton, ${ }^{[a]}$ \\ Maria Vallet-Regi, ${ }^{[c, d]}$ Daniel Ruiz-Molina, ${ }^{[b]}$ and Samuel Sánchez ${ }^{[a, e, f]}$
}

Abstract: In the field of micromotors many efforts have been taken to find a substitute for peroxide as fuel. While most approaches turn towards other toxic high energy chemicals such as hydrazine, we introduce here an energy source that is widely used in nature: light. Light is an ideal source of energy and some materials, such as $\mathrm{AgCl}$, have the inherent property to transform light energy for chemical processes, which can be used to achieve propulsion. In the case of silver chloride, one process observed after light exposure is surface modification, which leads to the release of ions, generating chemo-osmotic gradients. Here we present endeavors to use those processes to propel uniquely shaped micro-objects of microstar morphology with a high surfaceto-volume ratio, study their dynamics and present approaches to go towards real environmental applications.

\section{Introduction}

Continuous energy conversion is required to propel micromotors. The most commonly employed method used with this aim is the conversion of chemical energy from the degradation of high energy chemicals such as peroxide ${ }^{[1]}$ or hydrazine. ${ }^{[2]}$ However, many efforts are taken to find alternatives to these toxic components whenever applications such as biomedical or environmental remediation applications are envisaged..$^{[3]}$ Among them, enzymes open possibilities for more biocompat-

[a] Dr. J. Simmchen, A. Miguel-Lopez, Dr. M. M. Stanton, Dr. S. Sánchez Smart NanoBioSystems

Max-Planck-Institut für Intelligente Systeme Heisenbergstr. 3, 70569 Stuttgart (Germany)

E-mail: juliane.simmchen@tu-dresden.de

[b] Dr. J. Simmchen, Dr. D. Ruiz-Molina

Catalan Institute of Nanoscience and Nanotechnology (ICN2)

CSIC

and The Barcelona Institute of Science and Technology Campus UAB, Bellaterra, 08193 Barcelona (Spain)

[c] Dr. A. Baeza, Prof. Dr. M. Vallet-Regi

Dep. de Química InorgánicayBioinorgánica UCM

Plaza Ramón y Cajal s/n, Madrid (Spain)

[d] Dr. A. Baeza, Prof. Dr. M. Vallet-Regi

Centro de Investigación Biomédica en Red de Bioingeniería, BiomaterialesyNanomedicina (CIBER-BBN)

(Spain)

[e] Dr. S. Sánchez

Institució Catalana de Recerca i Estudis Avancats (ICREA), Pg.

Lluís Companys 23, 08010, Barcelona (Spain)

[f] A. Miguel-Lopez, Dr. S. Sánchez

Institut de Bioenginyeria de Catalunya (IBEC)

Baldiri I Reixac 10-12, 08028 Barcelona (Spain) —Please check addresses match authors, particularly $[f]$

$\square$ Supporting information for this article can be found under: http://dx.doi.org/10.1002/cnma.201600300. ible fuels, ${ }^{[4]}$ and alternatives routes such as surface-tensiondriven propulsion due to the release of solvent have been investigated..$^{[5]}$ Light is an ideal source of energy and some materials are able to transform this energy directly by undergoing chemical changes so that no toxic chemicals are required. ${ }^{[6]}$ For instance, Palacci et al. achieved peroxide degradation by hematite under light irradiation, ${ }^{[7]}$ and peroxide-free approaches have been presented, for example, on $\mathrm{TiO}_{2}$ structures $^{[8]}$ but the motion mechanism is not yet completely understood. The collective motion of the $\mathrm{AgCl}$ particles by UV light has also been studied. ${ }^{[9]}$ Light exposure induces a surface modification of the particles due to a state change from ionized to metallic silver that creates a localized electrolyte gradient around the particle that results in self-diffusiophoresis. ${ }^{[10]}$ The hypothesized degradation of $\mathrm{AgCl}$ is shown in Equation (1).

$4 \mathrm{AgCl}+2 \mathrm{H}_{2} \mathrm{O} \stackrel{\text { UV- light }}{\longrightarrow} 4 \mathrm{Ag}+4 \mathrm{H}^{+}+4 \mathrm{Cl}^{-}+\mathrm{O}_{2}$

Ibele et al. found that $\mathrm{AgCl}$ particles with asymmetric shape move autonomously in deionized (DI) water when exposed to UV light. ${ }^{[9 c]} \mathrm{A}$ more recent work analyses the motion patterns of silver chloride particles under UV light classifying them ac cording to the interaction between neighboring particles as isolated, coupled or schooled particles. ${ }^{\text {[a] }}$

Herein we describe the synthesis and study of new $\mathrm{AgCl}$ micromotors with novel microstar-shaped morphologies. Branched microstructures were obtained with a high surfaceto-volume ratio, which is expected to facilitate the motion of the resulting structures due to the higher number of active sites. We show how these motors exhibit three different motion modes in the presence of UV, including translational, rocking, and rotational motion, which were quantified. We also 
demonstrate applications based on the intrinsic properties of $\mathrm{AgCl}$, such as catalytic degradation of organic molecules and bacteriostatic effects.

\section{Results and Discussion}

\section{Synthesis}

Obtaining branched microstructures with a high surface-tovolume ratio is a challenge and here we achieved it by tuning crystal growth kinetic factors with the concentration of $\mathrm{Cl}^{-}$ ions in solution (see details in Supporting Information). Using a simple approach to control the release of $\mathrm{Cl}^{-}$ions in solution we engineer the formation of hierarchically branched superstructures. The silver chloride precipitates as by-product in a reaction to produce supermolecular structures (as can be seen Scheme 1).

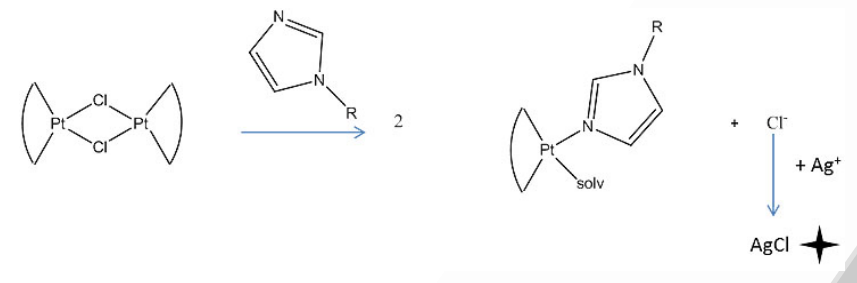

Scheme 1. Schematic representation of the synthetic strategy followed to obtain microstar-shaped $\mathrm{AgCl}$ particles.

In a typical experiment, $\mathrm{AgPF}_{6}$ is added to a solution of the platinum complex $\left[\mathrm{Pt}(\mu-\mathrm{Cl})_{2}(\mathrm{dmba})_{2}\right]\left(\mathrm{dmba}=\left(N, N^{\prime}\right.\right.$-dimethylbenzylamine) पok? in DMSO in which no precipitate is observed (structure shown in Supporting Information). Upon addition of a heterocyclic compound, delayed precipitation of $\mathrm{AgCl}$ occurred from co-formation and dissolution. We added the given ligand in order to shift the equilibrium towards that of the desired $\mathrm{AgCl}$ structures. D previous two sentences ok? Upon reaction with compound 2 (see Scheme 1), the controlled release of the $\mathrm{Cl}^{-}$ions and the subsequent precipitation with $\mathrm{Ag}^{+}$present in solution was induced. The supermolecular structures remain in solution, and $\mathrm{AgCl}$ precipitates were isolated by centrifugation.

The chemical composition of the precipitate was confirmed by EDX analysis, as shown in Figure $1 \mathrm{a}$. The shape is tuneable by adjusting the amount of free $\mathrm{Cl}^{-}$ions by the addition of a heterocyclic compound, resulting in cube-like structures or overgrown octapod-shaped microstar structures $(\mu S)$ Figure $1(\mathrm{~b}-\mathrm{e})$. At low $\mathrm{Cl}^{-}$concentrations, the cubic seeds precipitate showing already an incipient preferential growth through the cube edges (image not shown). As the ratio is increased, the concave cubes grow larger along the directions of corners up to the formation of microstars $(\mu \mathrm{S})$. These results are associated with the two different stages involved in crystallization: nucleation and growth. The preferential overgrowth required to obtain $\mu \mathrm{S}$ is due to different energy content of atoms at corners and edges. ${ }^{[11]}$ An increase above 1.0 equivalents results
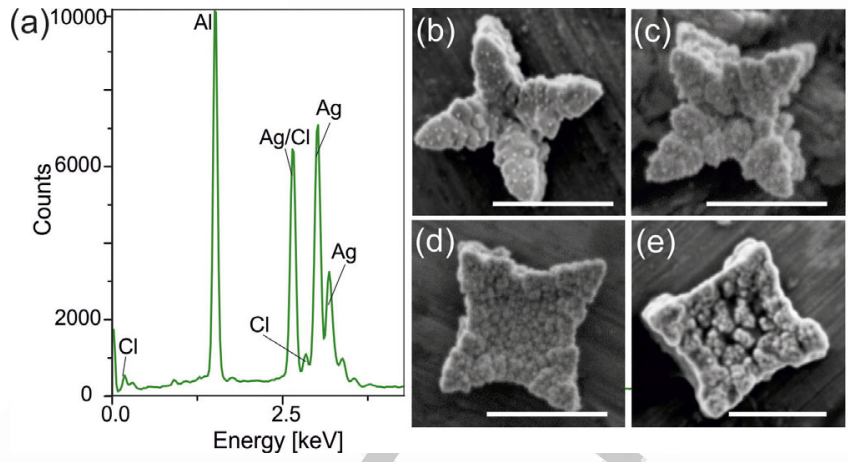

Figure 1. Synthesis of AgCl microstars. a) EDX analysis of the precipitate. All signals can be associated either to silver or to chloride, only the obtained Al-signal is due to the aluminum sample holder. b-e) The morphology of the $\mathrm{AgCl}$ precipitate strongly depends on the stoichiometry of heterocycles added (while keeping constant the $1: 1 \mathrm{Ag} / \mathrm{Cl}$ ratio): from $\mu \mathrm{S}$ structures to concave cubes with overgrown corners, the average end-to-end dimensions were about $2 \mu \mathrm{m}$. Scale bars equal $1 \mu \mathrm{m}$.

in the formation of cubic $\mathrm{AgCl}$ microstructures. This preferential growth increases upon increasing the $\mathrm{Cl}^{-}$concentration eventually leading to the formation of the octapod structures. Finally, at higher concentrations, the availability of $\mathrm{Cl}^{-}$is so high that less selective "docking" occurs and the energy differences between the different crystal facets lose importance. Other product/solvent combinations lead to partial precipitation, but no special shapes were observed (see Figure S1, Supporting Information).

\section{Dynamics of motion}

$\mathrm{AgCl}$ microstars gain motility one by one after irradiation with focused UV light from a mercury arc lamp at maximum intensity $(120 \mathrm{~W}$, DAPI excitation $340-380 \mathrm{~nm}$ ) (see Figure S3, Supporting Information). This can be explained by the gradient build up after the onset of light, triggering the particle motility. Those active particles undergo changes on their surface, discharging chloride ions and creating the chemical gradient to propel the particle motion. Gradient-driven motion has been discussed for motile particles mostly in the context of oxygen gradients that lead to propulsion, but other forms, such as ion gradients, have also been proposed. ${ }^{[10,12]}$ Here, the gradient is caused by the released chloride ions upon irradiation of $\mathrm{AgCl}$ structures with UV light from a mercury arc lamp at maximum intensity. The hypothesis that a chloride ion gradient is responsible for the motion is underpinned by the observation that in high concentrations of chloride ions (such as $0.1 \mathrm{M} \mathrm{KCl}$ ) UV light caused no motion.

Comparing both shapes we observed that smaller cubes rotate much faster, which is counterintuitive at first because the reaction of $\mathrm{AgCl}$ with light is a surface reaction; therefore, an increase in surface area should lead to an absolute increase of created product. Assuming a constant volume for both cubes and $\mu \mathrm{Ss}$, we calculated the volume/surface ratio and found, that the $\mu \mathrm{S}$ has a 1.7 times larger surface area (see Table 1). Even though the amount of product created by a larger surface may be increased, the distribution of the prod- 
Table 1. Geometric properties of an exemplary cube vs. microstar ( $\mu \mathrm{S})$ of $1 \mu \mathrm{m}^{3}$ volume.

\begin{tabular}{|lll|} 
& Cube & $\mu \mathrm{S}$ \\
\hline Volume $(V)$ & $1 \mu \mathrm{m}^{3}$ & $1 \mu \mathrm{m}^{3}$ \\
Side length $(a)$ & $1 \mu \mathrm{m}$ & $1.26 \mu \mathrm{m}$ \\
Surface $(A)$ & $6 \mu \mathrm{m}^{2}$ & $10.64 \mu \mathrm{m}^{2}$ \\
Ratio $A / V$ & 6 & 10.64 \\
\hline
\end{tabular}

uct over a larger area and the changes in angular momentum cause the $\mu \mathrm{Ss}$ to turn more steadily. Their different angular momentums are associated with the quadratic dependence of the distance between extremes from the central axis, and secondly, a larger surface also means a larger interaction interface with the surroundings. From now on further studies to gain more insight on the dynamics of $\mathrm{AgCl}$ were exclusively concentrated on $\mu \mathrm{Ss}$. This is due to their larger structure and therefore easier detection and tracking. Ballistic motion in one constant direction is rarely observed: most of the time diffusive (Figure $2 \mathrm{a}$ ) and rotational (Figure $2 \mathrm{~b}$ ) motions were observed simultaneously.

The path of an individual particle is also highly dependent on other external influences such overlapping gradients due to the presence of adjacent particles. This confirms earlier findings of Sen's group where isolated particles showed behavior similar to Brownian motion while coupled particles exhibited significantly higher diffusion constants. ${ }^{[13]}$ The translational component is characterized by plotting the different mean square displacements (MSD, for details on the formula see the Experimental Section) averaged for three different $\mu \mathrm{S}$ samples.
The results are shown in Figure 2. As can be seen there, $\mu S$ exhibit a relatively straight directed motion (III) with a curved MSD over $10 \mathrm{~s}$, while the other more randomly moving stars (I\&II) show a much less curved MSD behavior. Similar observations can be made for the rotational component. The analysis of two differently rotating stars is presented in Figure $2 \mathrm{~b}$. The upper star is rotating back and forth, resulting in a non-constant "continuous angle change" ( $\Delta \theta$, green curve) that reflects the increasing and diminishing of the angle respective to an initial position. A different way to characterize this motion is the "mean square angular displacement" (MSAD, equivalent to MSD for angular changes, formula in Supporting Information) which is found to be an almost straight line. On the contrary, a star performing constant rotation over a prolonged time frame is shown in red (Figure $2 b$ ). $\square$ ok? $\square$ This type of rotation is characterized by a constantly increasing $\Delta \theta$ because the star is never turning backwards, which translates into a parabolic MSAD. Depending on the inherent properties of each star and its surroundings, we find different modes of motion.

\section{Durability of motion}

Considering that the mechanism powering the motion involves the slow transformation of the particle itself, additional experiments were done to determine the duration. To do so, an aqueous colloidal dispersion of the $\mu \mathrm{S}$ was placed in an inverted microscope under UV-light illumination within a flat petri dish covered to inhibit solvent evaporation and the movement was followed over time. Every 60 minutes (up to three hours), videos of particle movement were taken and evaluated. With progressing time the catalytic surface reaction modifies the (a)

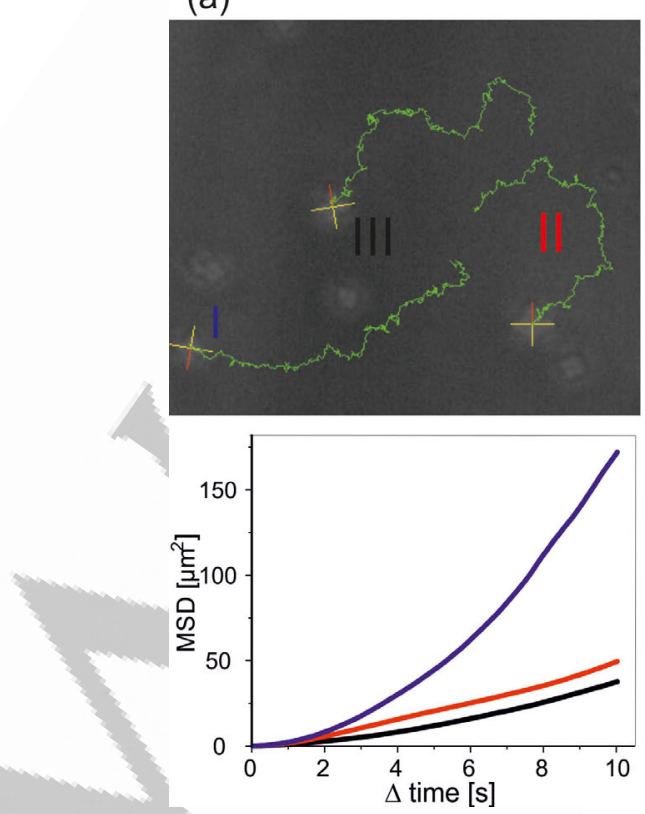

(b)
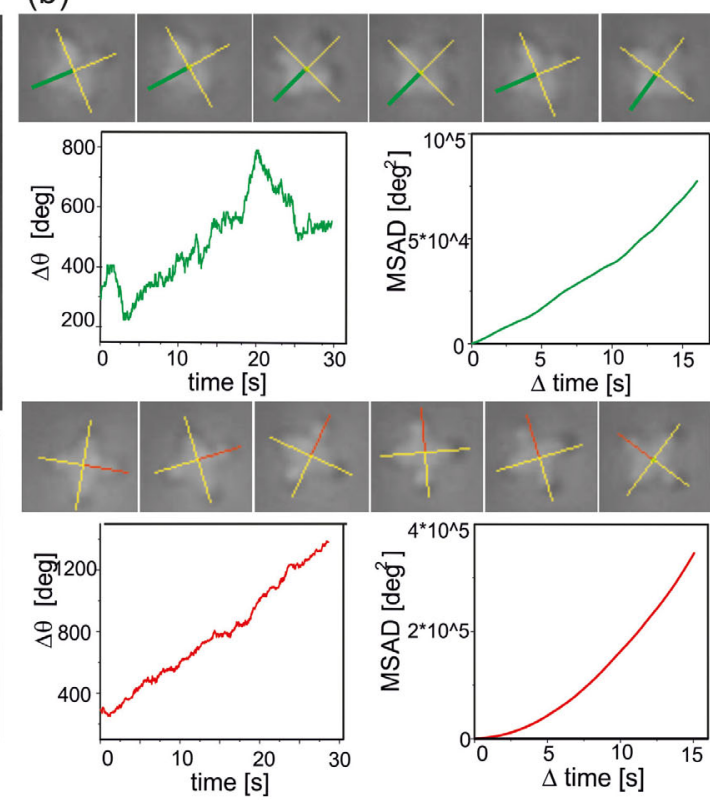

Figure 2. a) Translational component of motion-tracked enhanced Brownian diffusion of three motile AgCl $\mu$ Ss and the respective MSDs (blue line star l, red line star II, black line star III). b) Within the movement of a single microstar, the rotational parts were tracked and analyzed, two different kinds of rotation are displayed: the green back and forth rotating star results in a much less curved, almost straight MSAD while the red star constantly rotating in one direction results in a more curved MSAD. 

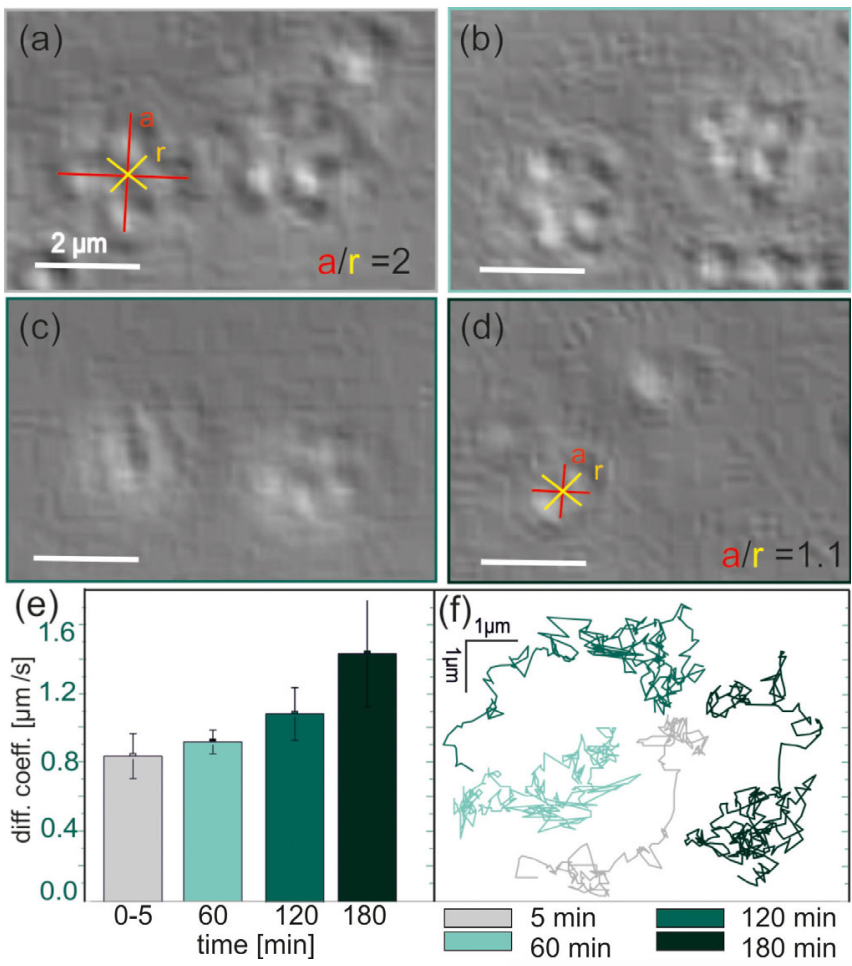

Figure 3. Lifetime and motion of light-activated $\mu \mathrm{S}$. a-d) shape of the $\mu \mathrm{S}$ at different times: a) 5, b) 60, c) 120 , d) $180 \mathrm{~min}$. The images show the subsequent degradation of the $\mu \mathrm{S}$ structures due to surface modification. Ratio of arm length versus radius indicates the degradation of the $\mu S$ with time. e) Speeds obtained from the MSD of minimum $3 \mu \mathrm{S}$ at different times. f) Trajectories of exemplary $\mu \mathrm{S}$, each track corresponds to $10 \mathrm{~s}$ of $\mu \mathrm{S}$ motion.

(a)

(b)
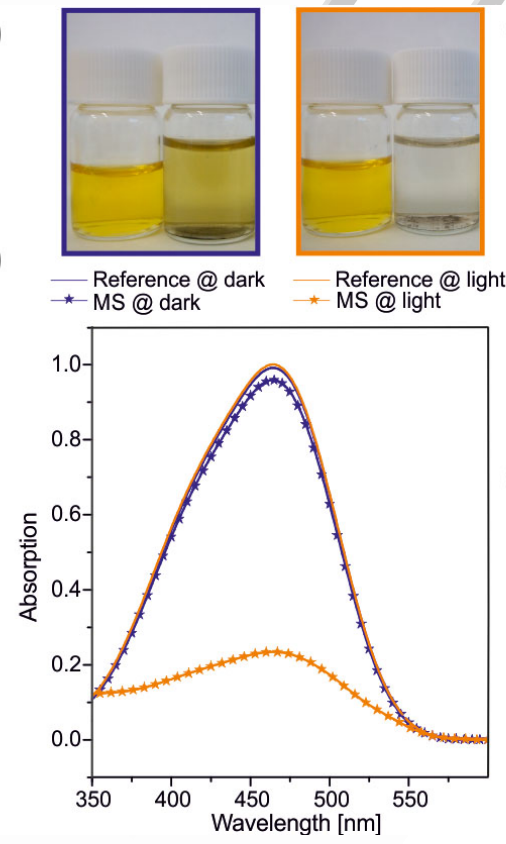

(d)
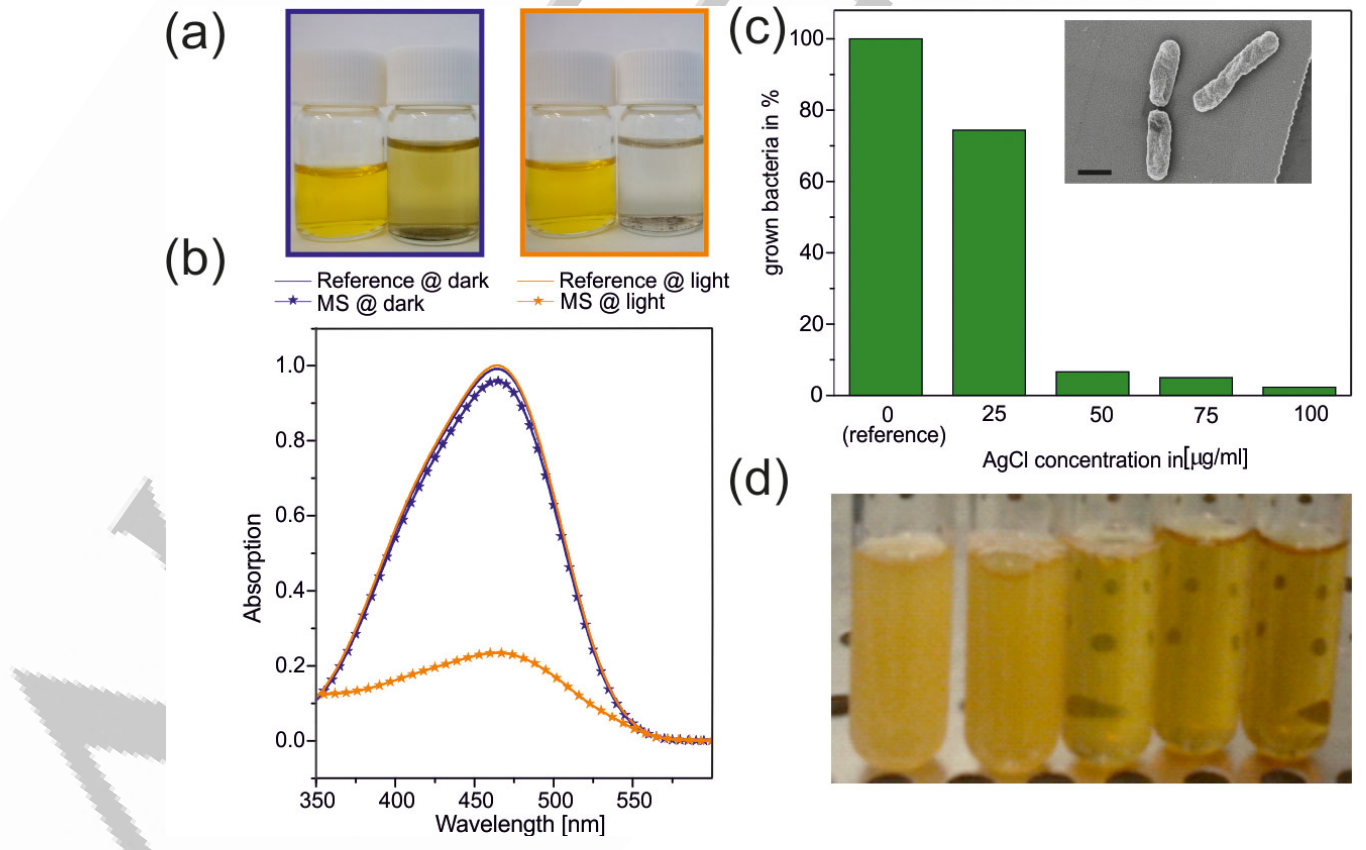

Figure 4. Environmental applications of $\mu \mathrm{S}$ : a-b) The photocatalytic activity of $\mathrm{AgCl} \mu \mathrm{S}$ was evaluated by testing the photocatalytic degradation of methyl orange (MO) aqueous solution. a) Optical image after $3 \mathrm{~h}$ of irradiation; the solutions exposed to sun were almost completely colorless. The reference samples help evaluating the stability of the molecules exposed after light exposure but in absence of $\mathrm{AgCl}$; it can be clearly seen that only in presence of both $\mathrm{AgCl}$ and light can the decomposition of the dye take place. b) Absorption spectrum of the MO samples and blank solutions. c) Inhibition of bacterial growth tested on $E$. coli, values measured by densitometry. Inset: SEM image of $E$. coli bacteria, scale bar corresponds to $1 \mu \mathrm{m}$. d) Optical evaluation of bacterial growth in solution. At about $50 \mu \mathrm{g} \mathrm{m}^{-1}$ a threshold concentration is reached that inhibits bacterial growth. particle shape, from $\mu \mathrm{S}$ (see inset $a / r$ in Figure 3 a $\mathbf{D}$ please define variables $\square$ ) to roundish body particles resulting in the observation of mainly stubs after long periods of time (see Figure $3 \mathrm{~d}$ ).

Nevertheless, the formation of stubs after exposition for $3 \mathrm{~h}$ does not disrupt motion, as can be seen in the exemplary motion of the different stages shown in Figure $3 \mathrm{e}-\mathrm{f}$. The trajectories of the corresponding particles after 5, 60, 120 and $180 \mathrm{~min}$ are displayed in Figure $3 \mathrm{f}$ ). The bars in Figure $3 \mathrm{e}$ show the different translational components of the motion. Their MSD evaluation resulted in effective diffusion coefficients, where a higher value correlates to a higher velocity. In the absence of light, particles can be stored in water for a several days without any significant morphology modification; after drying, storage was possible over month without losing shape. In dry state the only observed change was nanograin formation on the surface.

\section{Potential environmental applications}

Catalytic micromotors can be exceptional devices for environmental application ${ }^{[14]}$ by taking advantage of the spontaneous movement. In the present work, two environmental applications inherently related to the properties of $\mathrm{AgCl}$ were explored: I) degrading organic material ${ }^{[15]}$ such as certain pollutants ${ }^{[16]}$ through photocatalytic properties and II) antibacterial treatment with silver, which is widely known for its antibacterial properties, as recently demonstrated by Pané's group with silver-coated helical nanomachines. ${ }^{[17]}$ aok? $\mathbf{\square}$ The photocatalytic activity of $\mathrm{AgCl} \mu \mathrm{S}$ was evaluated with an aqueous so- 
lution of methyl orange (see Figure 4a-b). Specifically, two different solutions with identical concentrations of dyes were prepared, with and without $\mu \mathrm{Ss}$, and were exposed to sunlight irradiation while the evolution of the organic dye was followed by UV/Vis absorption spectroscopy. Two additional solutions were also prepared and kept in the absence of any light source for comparison purposes. After $3 \mathrm{~h}$ stored in darkness none of the two controls showed any significant change (blue framed image in Figure $4 \mathrm{a}$ and blue curves in b). Similar results were obtained for the sample exposed to light but in the absence of $\mu \mathrm{Ss}$. Finally, the solution containing the $\mu \mathrm{Ss}$ and exposed to sunlight was found to be almost completely colorless (right sample, orange framed image Figure $4 a$, orange line with star symbols in b). To prove the versatility of the catalytic decomposition on different chemical structures this study was extended to methyl blue and rhodamine $6 \mathrm{G}$ with similar excellent results (for more details see Figure $S 5$ in the Supporting Information).

A second set of experiments was designed and performed to demonstrate the antibacterial properties of $\mathrm{AgCl} \mu \mathrm{S}$. As can be seen in Figure 4, after addition of $25 \mu \mathrm{g} \mathrm{mL}^{-1} \mu \mathrm{S}$ the bacteria growth dropped by approximately $25 \%$ (confirmed by densitometry and colony counting, for details see Experimental Section). Further increase of the $\mu \mathrm{S}$ concentration to $50 \mu \mathrm{g} \mathrm{mL}^{-1}$ inhibited the growth of E. coli drastically: only $6.6 \%$ of the growth was found compared to the reference sample. Further increase of the $\mathrm{AgCl}$ concentration hardly lowered the growth rate any further (values obtained by densitometry and colony counting confirmed those results, further details in the Supporting Information). To elucidate the mechanism of these antibacterial properties the influence of the state of the $\mu \mathrm{Ss}$ (fresh or aged, and presence or absence of light) was tested, but caused almost no variation in the results. This observation leads to the conclusion that the photocatalytic processes play only a minor role for the bacteriostatic effect and the main factor seems to be the concentration of silver ions.

These observations led to the hypothesis that there is a maximum amount of soluble ion concentration in the Todd Hewitt broth (THB) medium, which is reached around $50 \mu \mathrm{g} \mathrm{mL}^{-1}$. To verify this hypothesis the concentration of $\mathrm{Ag}^{+}$ions in $\mathrm{THB}$ was measured by ICP-MS and compared to a corresponding inorganic buffer (see Supporting Information, Figure S6). The solubility of $\mathrm{AgCl}$ in salt solution and THB differs strongly, probably due interactions with the organic components of THB such as complexation phenomena.

\section{Conclusions}

We have demonstrated a novel synthesis route for uniquely shaped $\mathrm{AgCl}$ particles and their ability to be used as lightdriven micromotors. The manufacturing is based on delayed a precipitation that allows the control of precipitation dynamics and therefore offers a possibility to obtain a range of shapes from cubes and to octapod-shaped microstars. Those microstars enable us to observe the motion in pure water after UV irradiation and characterize three different modes: translation, rotation and rocking. To reach one step further we showed as a proof of concept that the $\mathrm{AgCl}$ microstars have potential to be applied in waste water treatment due to their versatile photocatalytic activity enabling them to degrade organic molecules. Another promising feature for waste water treatment is their bacteriostatic properties, which lead to an inhibition of bacterial growth. In conclusion, we consider that these findings broaden our knowledge of UV-driven micromotors and the novel shapes open possibilities to study shape-dependent collective behavior. However, further efforts are needed to bring this new kind of motors to the level of control that can be reached currently with other types of micromotors, such as directionality and speed control.

\section{Experimental Section}

\section{Experimental Details}

\section{Synthesis of $\mathrm{AgCl}$ particles}

In a typical synthesis $\left[\mathrm{Pt}(\mu-\mathrm{Cl})_{2}(\mathrm{dmba})_{2}\right](10 \mathrm{mg}, 0.0133 \mathrm{mmol})$ was dissolved in $4 \mathrm{~mL}$ DMSO. A solution of 1 equiv of an inorganic silver salt $\left(\mathrm{AgPF}_{6}\right)$ in $1 \mathrm{~mL}$ DMSO was added dropwise and stirred for $1 \mathrm{~h}$ protected from light. Initially no precipitation of $\mathrm{AgCl}$ was observed. In order to shift the equilibrium towards the products 1 equiv of bis(imidazol---ylmethyl)benzene or 2 equiv of imidazole were added and enhanced precipitation of $\mathrm{AgCl}$ was observed. The precipitate was filtered and washed with $\mathrm{EtOH}$.

1,4-Bis(imidazol-l-ylmethyl)benzene (bix) was synthesized following a method published by P.K. Dhal et al.

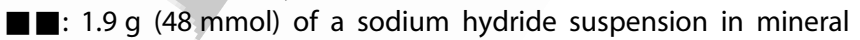
oil $(60 \%)$ were washed with $10 \mathrm{~mL}$ of dry THF under nitrogen and subsequently dissolved in $30 \mathrm{~mL}$ dry THF. Under a nitrogen atmosphere, imidazole $(2.85 \mathrm{~g}, 44 \mathrm{mmol})$ dissolved in $15 \mathrm{~mL}$ of dry THF was added slowly and the mixture was allowed to stir for $30 \mathrm{~min}$. a, $\mathrm{a}^{\prime}$-Dibromo- $p$-xylene $(5.3 \mathrm{~g}, 20 \mathrm{mmol})$ in $20 \mathrm{~mL}$ of THF was added to the resulting suspension, which was then heated to $50^{\circ} \mathrm{C}$ for minimum of $4 \mathrm{~h}$. The reaction mixture was cooled with approximately $25 \mathrm{~mL}$ of ice water and stirred for $20 \mathrm{~min}$. Then the product was extracted with chloroform $(3 \times 50 \mathrm{~mL})$, and the combined organic phases were dried over anhydrous sodium sulfate. After filtration, the solvent was removed under reduced pressure and the obtained product was found to be pure in NMR analysis and used without further purification.

\section{UV driven micromotors}

Experiments were performed in a Zeiss Axiovert microscope coupled to an EXFO lamp (X-Cite) and either an AxioCam MRm or a Thorlab USB 2.0 camera. A sample holder bearing a freshly cleaned glass slide was covered by $100 \mu \mathrm{L}$ Milli-Q water and $2 \mu \mathrm{L}$ $\mu \mathrm{S}$ in water were added. When irradiated with white light generally no movement was observed, only after turning on the UV lamp (with DAPI filter) did the particles start to move. The particles continued their movement even after turning of the UV light for some time, indicating that the created gradient does not disappear by turning off the light.

\section{Degradation of organic molecules}

Microstars were precipitated as described previously, washed in ethanol and water and redispersed in DI water in a concentration of $0.01 \mathrm{mg} \mu \mathrm{L}^{-1}$. Solutions of methyl orange, methyl blue and rhod- 
amine $6 \mathrm{G}$ were prepared in a concentration of $10 \mathrm{mg} \mathrm{L}^{-1}$. Finally to $5 \mathrm{~mL}$ colorant solution $30 \mu \mathrm{L}$ of $\mu \mathrm{S}$ suspension were added, or, for blank experiments, $30 \mu \mathrm{L}$ of pure water. A blank solution and a $\mu \mathrm{S}-$ containing solution of each colorant were kept in the dark; equally, a blank solution and a $\mu \mathrm{S}$-containing solution without colorant

ok? were placed under a UV lamp and another batch of blank and test solutions were placed in the sun. The degradation was found to be incomplete after $30 \mathrm{~min}$, therefore the duration of the experiment was extended to $3 \mathrm{~h}$ when the solutions were either colorless or strongly decolorized. After that time, UV absorption was measured in the wavelength range corresponding to the colorant. Concentrations: catalyst concentration: $0.01 \mathrm{mg} \mathrm{\mu L}^{-1}$; solution of organic compounds: $10 \mathrm{mg} \mathrm{L}^{-1} ; 30 \mu \mathrm{L}$ of catalyst concentration was added to each sample. Absorptions were measured in the ranges (depending on the colorant) shown in Table 2.

\begin{tabular}{|lll|}
\hline \multicolumn{2}{|l|}{ Table 2. Absorption measurements. $\mathbf{\square}$ title ok? } \\
Organic colorant & Initial wavelength [nm] & Final wavelength [nm] \\
\hline Methyl orange & 250 & 600 \\
Methyl blue & 250 & 400 \\
Rhodamine 6G & 400 & 600 \\
\hline
\end{tabular}

\section{Bactericidal effect}

Several concentrations of silver nanoparticles $(0,25,50,75$ and $100 \mu \mathrm{g} \mathrm{mL}^{-1}$ ) were tested against E. coli. Therefore E. coli cells should always be done on freshly unfrozen cultures. DPlease clarify. Grown from? An agar plate was inoculated with $20 \mu \mathrm{L}$ of unfrozen glycerol stock and grown overnight. A single colony was used to inoculate $100 \mathrm{~mL}$ of THB (containing salts, dehydrated heart infusion, yeast enriched peptone and dextrose) solution and incubated at $37^{\circ} \mathrm{C}$ overnight. Density measurement gave 1.497 after $16 \mathrm{~h}$. A dilution 1:10 was found to have suitable density for exponential growth (0.322) and used for further experiments. Dilutions of the same solution $\left(10^{-1}\right.$ to $\left.10^{-6}\right)$ were inoculated on agar plates to count CFU, which resulted in $9.05 \times 10^{7} \mathrm{CFU} \mathrm{mL}^{-1}$. $\square$ superscript added ok here and below?

The interaction with silver nanoparticles was analyzed by adding silver nanoparticles to the E. coli solution of $9 \times 10^{7} \mathrm{CFU} \mathrm{mL}^{-1}$.

The homogeneous suspensions of $0-100 \mu \mathrm{g} \mathrm{mL}^{-1}$ were allowed to grow overnight under agitation in an orbital shaker to avoid particle sedimentation. Optical density was measured and CFUs were counted in dilutions of $10^{-2}$ and $10^{-4}$. To determine the light sensitivity the same protocol was followed, with the only difference that the growth occurred partially in darkness and partially under irradiation with a USB-LED lamp. To evaluate the amount of $\mathrm{AgCl}$ dissolved into solution, the concentration of $\mathrm{Ag}^{+}$ions in THB and pure inorganic buffer solution was measured. Therefore the synthesized $\mathrm{AgCl} \mu \mathrm{S}$ were added to $5 \mathrm{~mL}$ solution (THB or buffer, respectively) and shaken for $8 \mathrm{~h}$ before centrifugation to remove all not dissolved particles. Then samples were sent to ICP-MS analysis for $\mathrm{Ag}^{+}$ions.

\section{Tracking}

Following reference [18], accurate tracking of the nanoparticles was performed on the recorded videos to compute the mean square displacement (MSD) and mean square angular displacements (MSAD). To achieve statistical data tracking was done automatically employing an especially developed script in Python 2.7 using the OpenCV library. First the image frame is cleaned of noise by using different types of blurring (median, Gaussian and bilateral filtering, depending on the quality of the recording), and then a gradient is applied by convoluting with the appropriate sobel kernel, which approximates the first derivative at every pixel, using a finite differences method plus Gaussian smoothing. By thresholding the absolute of this gradient the contours of the $\mu \mathrm{S}$ can be obtained and the centers are then easily calculated. Bayesian decision-making (the closest shape to a previous position is always assumed to be the correct one) enables us to accurately follow the trajectory of the detected $\mu \mathrm{S}$. Then the MSD was approximated from the data points using Equation (2):

$\operatorname{MSD}[\Delta t]=\sum_{i=0}^{N-\Delta t} \frac{r\left[i+\overrightarrow{\Delta t}-\vec{r}[]^{2}\right.}{N-\Delta t}$

where $\Delta t$ is the time displacement, $N$ is the total number of data points, and $r[i]$ is the position at time $i$. The effective diffusion coefficient is obtained by fitting the data to Equation (3):

$\operatorname{MSD}[\tau]=4 D \tau$

which holds in this case because the movement is mainly enhanced Brownian motion. For the angle tracking a similar process is used: gradient analysis of the frames is also applied but with slightly different parameters, so that the arms of the stars are more clearly defined. By computing the four directions in which the contour is, on average, further away from the center, the four arms of every star can be distinguished at each frame, and the rotational movement tracked over time. The basic theory that describes the MSD in different types of translational motion can also be used to characterize rotational motion. A randomized spinning due to Brownian motion results in a linear mean square angular displacement, and a constantly changing angle trivially gives a parabolic shape. Using this magnitude makes it possible to easily characterize the different types of rotation. The equation is the same as for the MSD, but substituting position by angle.

\section{Acknowledgements}

All authors thank Jose Ruiz and Venancio Rodrıguez for providing the Pt-dimer and Lluis Soler for the help installing the camera. This project has been financially supported by the European Research Council (ERC) for Starting Grant 'Lab-in-a-tube and Nanorobotics biosensors; LT-NRBS' (no. 311529), the German Research Foundation (DFG, SA 2525/1-1). J.S. acknowledges the Jae-Pre fellowship from CSIC.

Keywords: antibacterial • environmental applications photoactive colloids $\cdot$ self-propellers $\cdot$ silver chloride

[1] a) W. F. Paxton, P. T. Baker, T. R. Kline, Y. Wang, T. E. Mallouk, A. Sen, J. Am. Chem. Soc. 2006, 128, $14881-14888$; b) D. Kagan, P. Calvo-Marzal, S. Balasubramanian, S. Sattayasamitsathit, K. M. Manesh, G.-U. Flechsig, J. Wang, J. Am. Chem. Soc. 2009, 131, 12082-12083; c) P. Calvo-Marzal K. M. Manesh, D. Kagan, S. Balasubramanian, M. Cardona, G.-U. Flechsig, J. Posner, J. Wang, Chem. Commun. 2009, 4509-4511; d) A. A. Solovev S. Sanchez, M. Pumera, Y. F. Mei, O. G. Schmidt, Adv. Funct. Mater. 2010 20, 2430-2435; e) L. Baraban, M. Tasinkevych, M. N. Popescu, S. Sanchez, S. Dietrich, O. G. Schmidt, Soft Matter 2012, 8, 48-52. 
[2] a) R. Laocharoensuk, J. Burdick, J. Wang, ACS Nano 2008, 2, 1069-1075; b) W. Gao, A. Pei, R. Dong, J. Wang, J. Am. Chem. Soc. 2014, 136, 2276 2279; c) M. E. Ibele, Y. Wang, T. R. Kline, T. E. Mallouk, A. Sen, J. Am. Chem. Soc. 2007, 129, 7762-7763.

[3] S. Tottori, L. Zhang, F. Qiu, K. K. Krawczyk, A. Franco-Obregón, B. J. Nelson, Adv. Mater. 2012, 24, 811-816.

[4] a) X. Ma, A. Jannasch, U.-R. Albrecht, K. Hahn, A. Miguel-López, E. Schäffer, S. Sánchez, Nano Lett. 2015, 15, 7043-7050; b) X. Ma, X. Wang, K Hahn, S. Sánchez, ACS Nano 2016, 10, 3597-3605; c) L. K. E. A. Abdelmohsen, M. Nijemeisland, G. M. Pawar, G.-J. A. Janssen, R. J. M. Nolte J. C. M. van Hest, D. A. Wilson, ACS Nano 2016, 10, 2652-2660; d) D. Walker, B. T. Käsdorf, H.-H. Jeong, O. Lieleg, P. Fischer, Sci. Adv. 2015, 1, e1500501.

[5] a) T. D. Frank, Condens. Matter Phys. 2014, 17, 43002; b) G. Zhao, M. Pumera, J. Phys. Chem. B 2012, 116, 10960-10963.

[6] M. Guix, C. C. Mayorga-Martinez, A. Merkoçi, Chem. Rev. 2014, 114 $6285-6322$

[7] J. Palacci, S. Sacanna, A. P. Steinberg, D. J. Pine, P. M. Chaikin, Science 2013, 339, 936-940.

[8] a) Y. Hong, M. Diaz, U. M. Córdova-Figueroa, A. Sen, Adv. Funct. Mater. 2010, 20, 1568-1576; b) S. Giudicatti, S. M. Marz, L. Soler, A. Madani, M. R. Jorgensen, S. Sanchez, O. G. Schmidt, J. Mater. Chem. C 2014, 2, $5892-5901$; c) M. Enachi, M. Guix, V. Postolache, V. Ciobanu, V. M. Fomin, O. G. Schmidt, I. Tiginyanu, Small 2016, 12, 5497-5505.

[9] a) W. Duan, M. Ibele, R. Liu, A. Sen, Eur. Phys. J. E 2012, 35, 77; b) M. E. Ibele, P. E. Lammert, V. H. Crespi, A. Sen, ACS Nano 2010, 4, 4845-4851; c) M. Ibele, T. E. Mallouk, A. Sen, Angew. Chem. Int. Ed. 2009, 48, 3308 3312; Angew. Chem. 2009, 121, 3358-3362.

[10] J. L. Anderson, Annu. Rev. Fluid Mech. 1989, 21, 61-99.

[11] H. Gatemala, C. Thammacharoen, S. Ekgasit, CrystEngComm 2014, 16, $6688-6696$.

[12] A. Brown, W. Poon, Soft Matter 2014, 10, 4016-4027.

[13] H. Wang, G. Zhao, M. Pumera, J. Am. Chem. Soc. 2014, 136, 2719-2722.

[14] a) L. Soler, S. Sanchez, Nanoscale 2014, 6, 7175-7182; b) M. Guix, J. Orozco, M. García, W. Gao, S. Sattayasamitsathit, A. Merkoçi, A. Escarpa J. Wang, ACS Nano 2012, 6, 4445-4451; c) W. Gao, A. Pei, J. Wang, ACS Nano 2012, 6, 8432-8438.

[15] a) C. An, R. Wang, S. Wang, X. Zhang, J. Mater. Chem. 2011, 21, 11532 11536 ; b) P. Wang, B. Huang, Z. Lou, X. Zhang, X. Qin, Y. Dai, Z. Zheng, X. Wang, Chem. Eur. J. 2010, 16, 538-544.

[16] F. Mushtaq, A. Asani, M. Hoop, X.-Z. Chen, D. Ahmed, B. J. Nelson, S. Pané, Adv. Funct. Mater. 2016, 26, 6995-7002.

[17] M. Hoop, Y. Shen, X.-Z. Chen, F. Mushtaq, L. M. Iuliano, M. S. Sakar, A. Petruska, M. J. Loessner, B. J. Nelson, S. Pané, Adv. Funct. Mater. 2016 26, $1063-1069$

[18] G. Dunderdale, S. Ebbens, P. Fairclough, J. Howse, Langmuir 2012, 28, 10997- 11006.

Manuscript received: October 17, 2016

Accepted Article published: November 21, 2016

Final Article published: $\square$ u, 0000 


\section{FULL PAPER}

\section{Micromotors}

Juliane Simmchen, * Alejandro Baeza, Albert Miguel-Lopez, Morgan M. Stanton, Maria Vallet-Regi, Daniel Ruiz-Molina, Samuel Sánchez

$\mathbf{\square - \square}$

Dynamics of Novel Photoactive AgCl Microstars and Their Environmental Applications
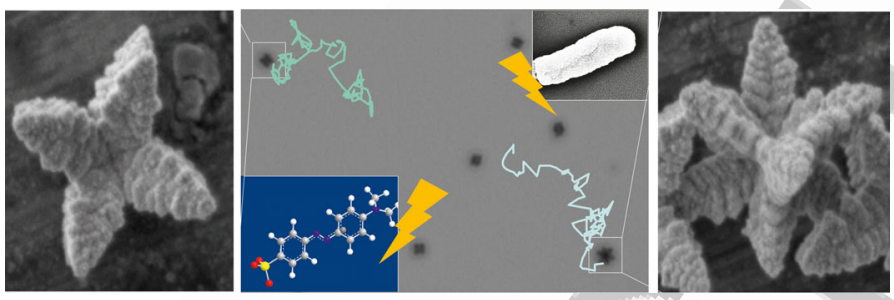

Please provide a short text for the

Self-propelling $\mathrm{AgCl}$ micromotors from J. Simmchen @MPI IS Tue SPACE RESERVED FOR IMAGE AND LINK

Share your work on social media! ChemNanoMat has added Twitter as a means to promote your article. Twitter is an online microblogging service that enables its users to send and read text-based messages of up to 140 characters, known as "tweets". Please check the pre-written tweet in the galley proofs for accuracy. Should you or your institute have a Twitter account, please let us know the appropriate username (i.e., @accountname), and we will do our best to include this information in the tweet. This tweet will be posted to the journal's Twitter account @ChemNanoMat (follow us!) upon online publication of your article, and we recommended you to repost ("retweet") it to alert other researchers about your publication.

Please check that the ORCID identifiers listed below are correct. We encourage all authors to provide an ORCID identifier for each coauthor. ORCID is a registry that provides researchers with a unique digital identifier. Some funding agencies recommend or even require the inclusion of ORCID IDs in all published articles, and authors should consult their funding agency guidelines for details. Registration is easy and free; for further information, see http:// orcid.org/.

Dr. Juliane Simmchen

Dr. Alejandro Baeza

Albert Miguel-Lopez

Dr. Morgan M. Stanton

Prof. Dr. Maria Vallet-Regi

Dr. Daniel Ruiz-Molina

Dr. Samuel Sánchez 